\title{
Electrochemical Characterization of Passive Films on Ni-Based Alloys in Acidic and Neutral Solutions
}

\author{
Whee Sung Kim*1, Hiroaki Tsuchiya and Shinji Fujimoto*2 \\ Division of Materials and Manufacturing Science, Graduate School of Engineering, Osaka University, \\ Suita 565-0871, Japan
}

\begin{abstract}
The present work reports the structure, chemical composition and electronic properties of passive films formed on Alloy 600 and Alloy 690 in borate buffer and sulfuric acid solutions. Photoelectrochemical response from passive films varied depending on the alloy substrates and the solutions. XPS analysis revealed that the passive films formed on Alloy 600 and Alloy 690 consisted of an outer hydroxide layer and an inner oxide layer in the borate buffer solution as well as the sulfuric acid solution. The chemical composition of the layers was relatively different depending on the solutions, that is, more Fe was incorporated into the passive films formed in the borate buffer solution.

[doi:10.2320/matertrans.M2014384]
\end{abstract}

(Received October 31, 2014; Accepted January 26, 2015; Published March 25, 2015)

Keywords: corrosion, passive film, nickel-based alloy, selective dissolution, photoelectrochemical response, X-ray photoelectron spectroscopy

\section{Introduction}

The corrosion resistance of metallic materials is attributed to passive films on their surface, that is, strongly depends on the structure, composition and properties of passive films. Therefore, the analysis of passive films has been considered to be prerequisite and carried out with various analytical techniques. Since the 1970's, the composition and structure of passive films have been investigated using the ex-situ analytical techniques such as XPS and AES. ${ }^{1-9)}$ Such ex-situ analytical techniques, however, denature passive films when analyzed, because any specimens should be taken out of solution and furthermore, measurements are performed in an ultra-high vacuum environment. Therefore passive films formed in aqueous solution have been examined also by in-situ techniques since the 1980 's. ${ }^{10-27)}$ In particular the photoelectrochemical response and the electrochemical impedance technique have often been used to examine passive films. The both in-situ analytical techniques provide information on electronic properties of passive films that are related with the stability of passive film. Therefore the in-situ techniques have been applied to examine electronic properties of passive film. The electronic properties of passive films on nickel-base alloys have been explored to reveal the high corrosion resistance of the alloys. ${ }^{18,23-25)}$ These analyses were based on Mott-Schottky plot from the electrochemical impedance measurement where type of conduction as semiconductor and carrier density in passive film can be estimated from the slope of Mott-Schottky plot. Although several slopes have been often observed in one MottSchottky plot and analyzed based on layered structure of passive film (hydroxide layer and oxide layer), it is not clear which part of passive film is analyzed from Mott-Schottky plot. On the other hand, the photoelectrochemical response measures the current change generated by photo-excitation in passive film. Assuming that the passive film consists of some layers along the substrate, and the each layer is constructed

\footnotetext{
${ }^{* 1}$ Graduate Student, Osaka University

${ }^{* 2}$ Corresponding author, E-mail: fujimoto@mat.eng.osaka-u.ac.jp
}

by a uniform composition, the photo-excitation is attributed to the band gap energy of each component in passive film. Moreover, values of band gap energies are different from each other on their compositions. Therefore, obtained data from the photoelectrochemical response can be separated into several components according to the band gap energy of each layer in passive film. Furthermore, from the potential dependence of photocurrent, the type of semiconductor can be also estimated. ${ }^{19,20)}$ These indicate that the photoelectrochemical response is a powerful in-situ technique to examine electronic properties of each layer in passive films. However, the electronic properties of passive films on $\mathrm{Ni}$ based alloys have not been investigated. Furthermore, the understanding of electronic properties of passive films on the alloys will lead to the development of high corrosion resistant alloy.

In the present work we have examined electronic properties of passive films formed on Alloy 600 and Alloy 690 in $\mathrm{pH} 8.4$ borate buffer and in $0.1 \mathrm{~mol} \mathrm{~L}^{-1}$ sulfuric acid solutions using the photoelectrochemical response. Especially how chemical composition of passive films affects electronic properties has been focused.

\section{Experimental}

The materials examined were Alloy 600, Alloy 690 and high-purity Alloy 690. The chemical compositions of the alloys are listed in Table 1. Furthermore, Ni-30Cr alloy was also used as a reference. The surfaces of the materials were polished mechanically with $\mathrm{SiC}$ abrasive paper and mirrorfinished with submicron alumina pastes, then cleaned with ethanol, methanol, and distilled water, successively.

Electrochemical measurements were carried out in a threeelectrode cell with a platinum counter electrode and a Ag/ $\mathrm{AgCl}$ reference electrode. The specimen electrode was connected to a lead wire by welding and then embedded in an epoxy resin. The specimen surface was mirror-finished with alumina paste, followed by ultra-sonicating in methanol and distilled water. The surface of the electrode was covered with polytetrafluoroethylene (PTFE) adhesive tape, leaving 
Table 1 Chemical compositions of specimens (mass\%).

\begin{tabular}{|c|c|c|c|c|c|c|c|c|c|c|c|c|c|}
\hline & $\mathrm{C}$ & $\mathrm{Si}$ & $\mathrm{Mn}$ & Co & $\mathrm{Ni}$ & $\mathrm{Cr}$ & $\mathrm{Fe}$ & Mo & $\mathrm{Ti}$ & $\mathrm{Al}$ & $\mathrm{P}$ & $\mathrm{S}$ & B \\
\hline Alloy 600 & 0.010 & 0.310 & 0.360 & - & 75.010 & 15.710 & 7.350 & - & - & - & 0.009 & $<0.001$ & - \\
\hline Alloy 690 & 0.020 & 0.120 & 0.260 & 0.030 & Bal & 29.550 & 9.610 & 0.020 & 0.110 & 0.90 & 0.0090 & 0.0020 & $<0.001$ \\
\hline High-purity Alloy 690 & 0.0019 & 0.01 & $<0.01$ & 0.02 & Bal & 28.92 & 10.0 & 0.01 & 0.09 & 0.09 & 0.0009 & 0.0008 & $<0.0001$ \\
\hline
\end{tabular}

$0.785 \mathrm{~cm}^{2}$ of the surface to electrolyte. The electrolytes used were $0.1 \mathrm{~mol} \mathrm{~L}^{-1}$ sulfuric acid and $\mathrm{pH} 8.4$ borate buffer solutions. Both were deaerated with $\mathrm{N}_{2}$ gas for more than $12 \mathrm{~h}$ prior to experiment. The polarization curves were measured in the solutions with a scan rate of $1 \mathrm{mV} \mathrm{s}^{-1}$. The electronic properties of passive films were investigated by the photoelectrochemical response. A specimen was polarized at a desired film formation potential, $E_{\mathrm{f}}$, in a passive region for $24 \mathrm{~h}$ before photoelectrochemical response measurements commenced. The photoelectrochemical response measurements were carried out using a potentiostat connected to a low-pass filter with a threshold frequency of $4 \mathrm{~Hz}$ and a differential amplifier. In the measurement, monochromatic light obtained using a 150-W xenon arc lamp and a grating monochromator was irradiated on the specimen in the electrochemical cell through a quartz window. The current change caused during the photoirradiation for $15 \mathrm{~s}$ was recorded as a photocurrent transient at the film formation potential, $E_{\mathrm{f}}$. Then the applied potential was changed stepwise with the interval of $100 \mathrm{mV}$ in the less noble direction. The measurement was carried out at each measuring potential, $E_{\mathrm{m}}$, changing the wavelength of the incident light from 250 to $500 \mathrm{~nm}$.

XPS analysis was performed to examine the chemical composition of passive films formed on the specimens in the solutions using a Rigaku XPS-7000 spectrometer. Al-K $\alpha$ $(250 \mathrm{~W})$ was used as X-ray source without monochromator. The spectrometer was operated with constant pass energy of $15 \mathrm{eV}$ and two different take-off angles of photoelectron were used to obtain the information on the structure of passive films on the Ni-based alloys. Obtained spectra were separated into those of some chemical states using the deconvolution software attached to XPS-7000 system and the Shirley method $^{28)}$ was applied for background subtraction. The composition of passive films was quantitatively estimated based on the integrated areas of the deconvoluted photoelectron spectra of $\mathrm{Ni}_{2} \mathrm{p}_{3 / 2}, \mathrm{Cr} 2 \mathrm{p}_{3 / 2}, \mathrm{Fe} 2 \mathrm{p}_{3 / 2}, \mathrm{O} 1 \mathrm{~s}$ and $\mathrm{C} 1 \mathrm{~s}$.

\section{Results}

Figures 1(a) and 1(b) show the potentiodynamic polarization curves of Alloy 600 and Alloy 690 in the borate buffer and sulfuric acid solutions, respectively. Polarization curves measured for Ni-30Cr alloy in the solutions are also included for comparison. It is clear that in the borate buffer solution, current density in the polarization curves was quite different among the specimens. However, the passive region was determined to be located between -400 and $400 \mathrm{mV}$ for all specimens. In the sulfuric acid solution, on the other hand, the polarization curves were more similar each other, and the passive region in the sulfuric acid solution was determined
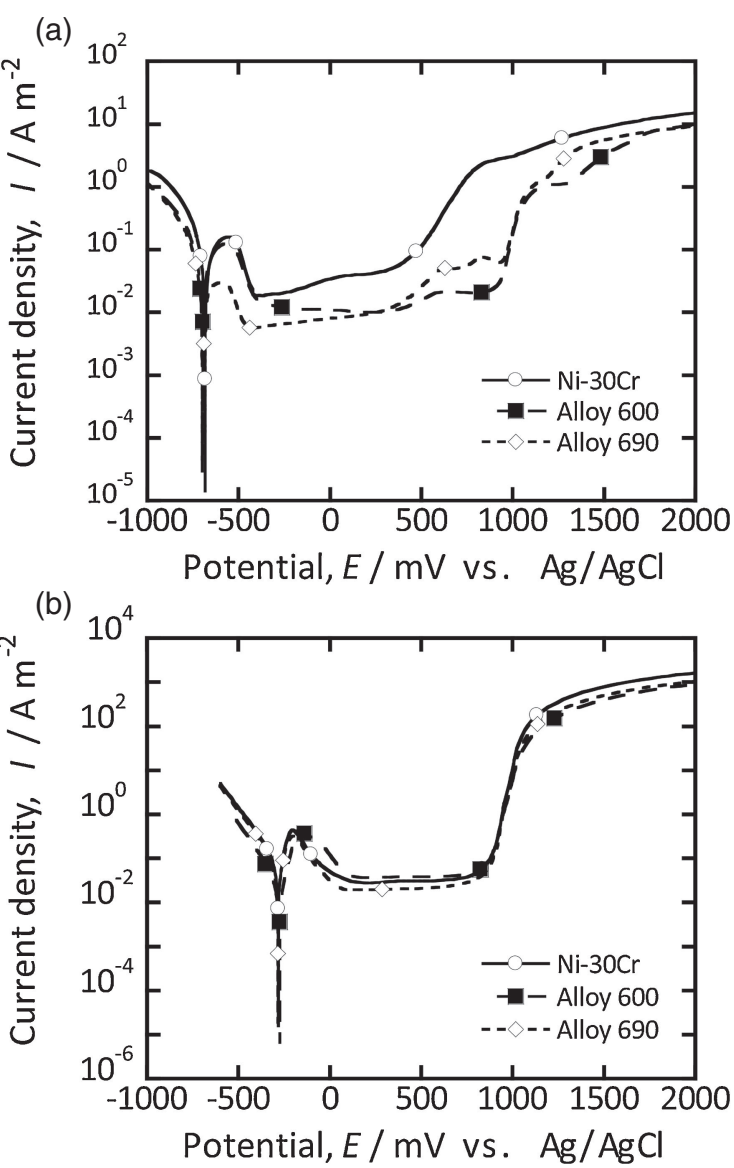

Fig. 1 Polarization curves of Alloy 600 and Alloy 690 measured in (a) the borate buffer solution and (b) the sulfuric acid solution. Polarization curve for $\mathrm{Ni}-30 \mathrm{Cr}$ is included as comparison.

to be from 100 to $900 \mathrm{mV}$. In the present work, photoelectrochemical response measurements were performed around the passive regions.

In the photoelectrochemical response measurements, current change caused by photoirraidation was recorded. The current change obtained in the present work exhibited three types of transient for all specimens in the solutions. Figure 2 shows current transients recorded for passive films formed on Alloy 690. At lower potentials, the shape of current transient was similar to that shown in Fig. 2(a) whereas at higher potentials, current exhibited the transients shown in Figs. 2(b) and 2(c) depending on the experimental condition. As apparent, the current is not constant, but varies during photoirradiation. Therefore, the difference between current recorded just before photoirradiation and after $15 \mathrm{~s}$ was defined as photocurrent, $i_{\mathrm{ph}}$, and analyzed in the present work. Figure 3(a) presents photocurrent spectra obtained at various measuring potentials for the passive film formed on 
(a)

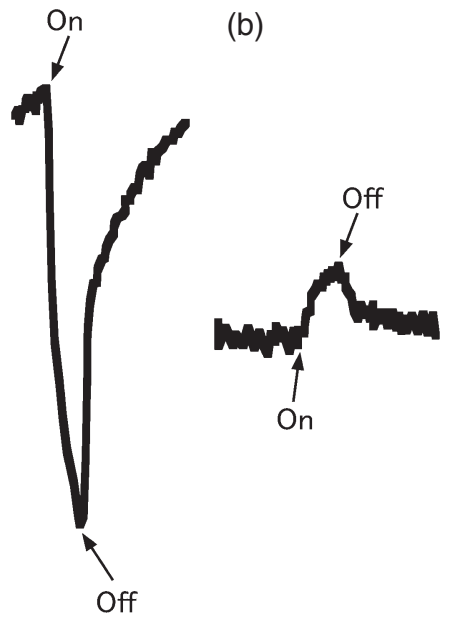

(c)

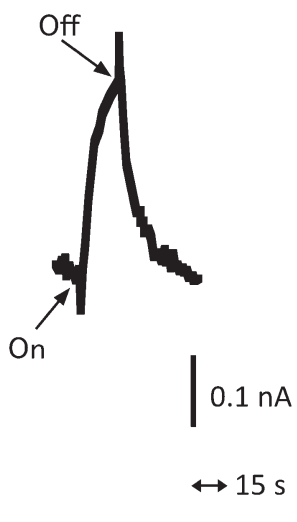

Fig. 2 Examples of photocurrent transient obtained for passive films formed on Alloy 690 in the sulfuric acid solution and the borate buffer solution. Experimental conditions were; (a) at $0 \mathrm{mV}$ in the sulfuric acid solution at the wavelength of $280 \mathrm{~nm}$, (b) at $100 \mathrm{mV}$ in the borate buffer solution at $290 \mathrm{~nm}$, (c) at $200 \mathrm{mV}$ in the borate buffer solution at $310 \mathrm{~nm}$.

Alloy 690 at $200 \mathrm{mV}$ in the borate buffer solution. It is clear that photocurrent varies depending on applied potential as well as the wavelength of incident light. As the intensity of the light is not constant for each wavelength, photocurrent spectra should be normalized for further analyses. Assuming that the photo excitation occurs as an indirect transition, photocurrent spectra can be normalized as photoelectrochemical action spectrum ${ }^{19-21)}$

$$
\left(i_{\mathrm{ph}} \cdot h v / I_{0}\right)^{\frac{1}{2}}=C\left(h v-E_{\mathrm{g}}\right)
$$

where $I_{0}$ and $h v$ are the intensity and the photon energy of incident light, respectively, and $E_{\mathrm{g}}$ is the band gap energy of passive film. $C$ is the slope of photoelectrochemical action spectrum and reflects the amplitude of photocurrent generated. The $h$ is the Planck's constant. By replotting the photocurrent spectra in Fig. 3(a), photoelectrochemical action spectra are obtained as shown in Fig. 3(b). As it is clear from the figure, the photoelectrochemical action spectra do not exhibit one straight line, but two regions with different slopes. Furthermore it is noticeable that the photocurrent spectrum obtained at $-100 \mathrm{mV}$ exhibits both positive and negative values depending on the photon energy of incident light. Assuming that photocurrent is generated from a single layer and the composition of one layer is uniform, the photocurrent should not change its direction at a fixed potential even if the photon energy of incident light is changed. Therefore, it can be considered that the photocurrent is generated from two layers. Angle-resolved XPS analysis revealed that passive films formed on the Ni-based alloys in the borate buffer and sulfuric acid solutions consist of duplex layers, that is, an inner oxide layer and a covering hydroxide layer. Typical spectra obtained for the passive film formed on Alloy 690 at $200 \mathrm{mV}$ in the borate buffer solution are shown in Fig. 4. The spectra show that the passive films formed on the Ni-based alloys consist of a hydroxide layer and an oxide layer. Furthermore angle-resolved XPS analysis (data are not shown) revealed that the hydroxide layer was present on the oxide layer. These indicate that the passive films on the alloys are composed of the outer hydroxide layer

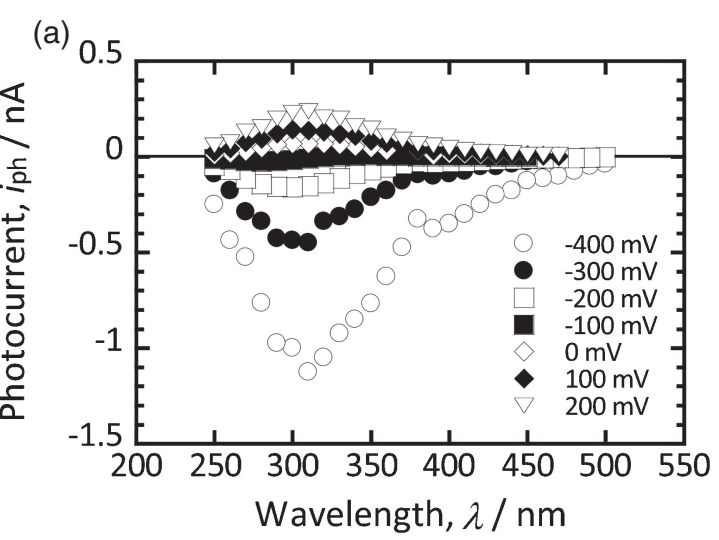

$\stackrel{7}{(b)}$

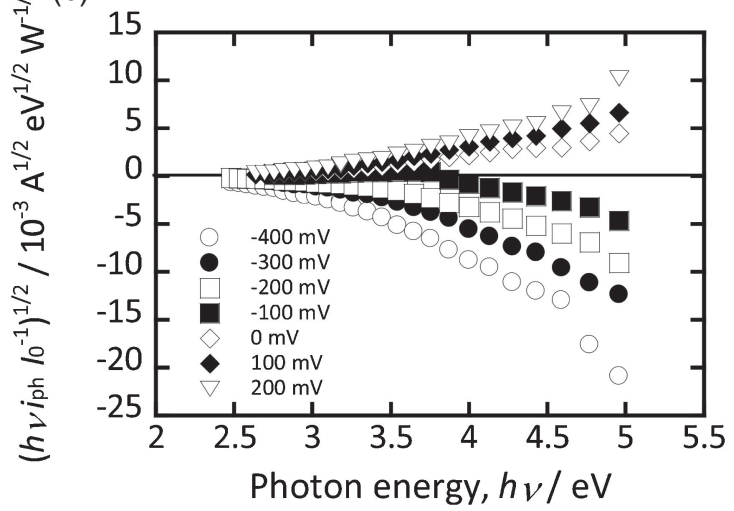

$\stackrel{7}{(c)}$

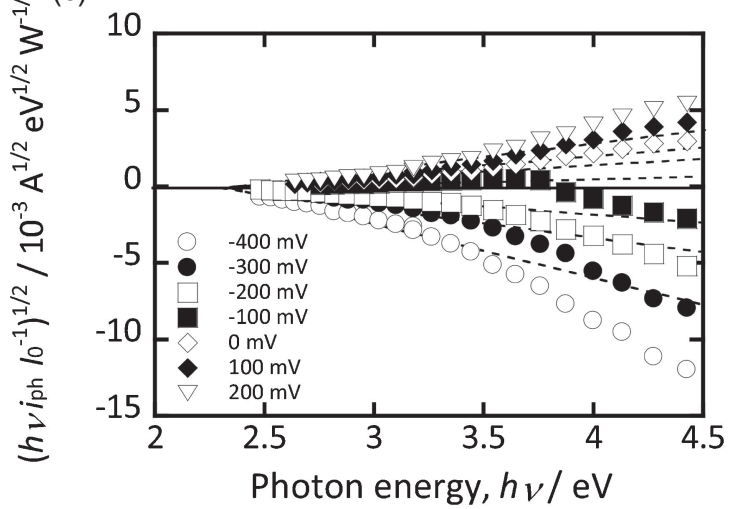

Fig. 3 (a) Photocurrent spectra for the passive film formed on Alloy 690 at $200 \mathrm{mV}$ in the borate buffer solution. The spectra were obtained at various measuring potentials. (b) Photoelectrochemical action spectra calculated from the photocurrent spectra shown in Fig. 3(a). (c) Magnified photoelectrochemical action spectra, shown in Fig. 3(b).

and the inner oxide layer. The XPS results obtained in the present work are in agreement with those previously reported in the literatures. ${ }^{5,6)}$ In the present work, therefore, the photoelectrochemical action spectra were separated into two components, assuming that photocurrent obtained for passive film is considered as sum of two components originated from inner oxide and outer hydroxide layers with different band gap energies. As a result, the band gap energy of a passive film and the slope of photoelectrochemical action spectrum, $C$, were obtained. Band gap energy, $E_{\mathrm{g}}$, is estimated as the photon energy at which the $\left(i_{\mathrm{ph}} \cdot h v / I_{0}\right)^{1 / 2}$ equals to zero in photoelectrochemical action spectrum. In the lower photon energy region, the photocurrent is generated from one layer with lower band gap energy. Therefore, the extrapolation of 
(a)

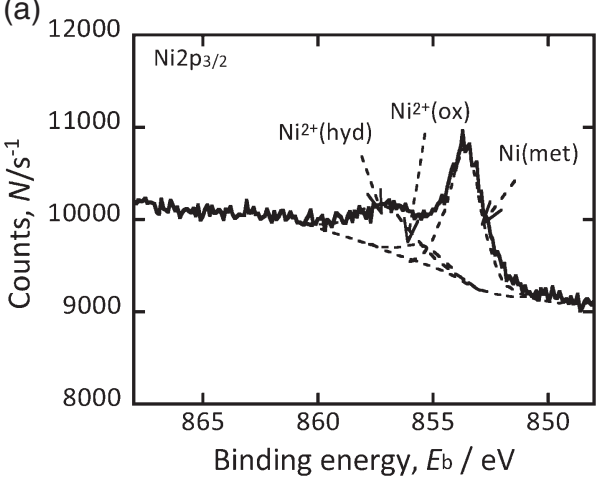

(c)

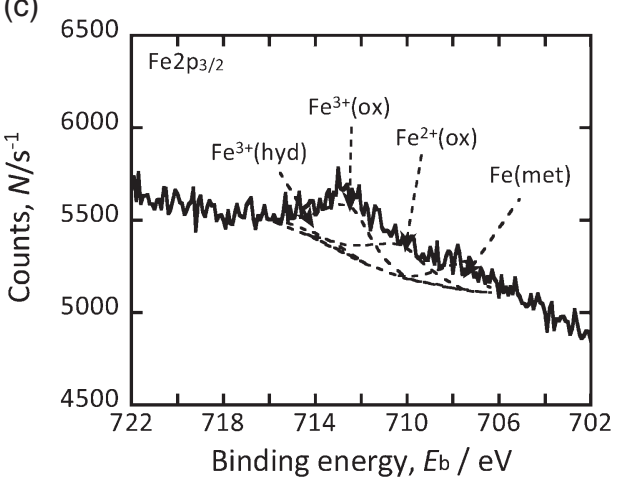

(b)

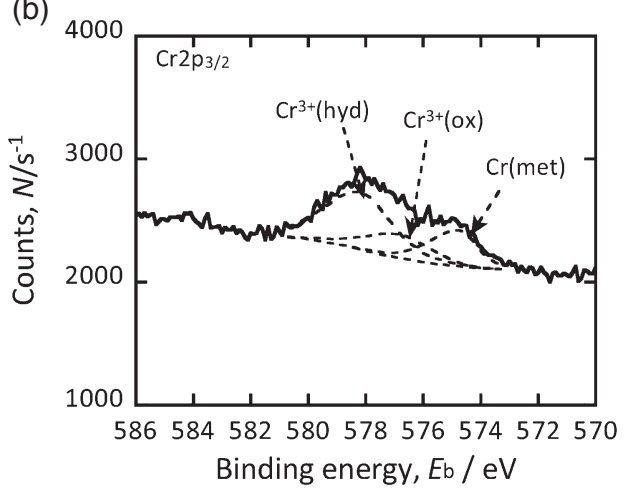

(d)

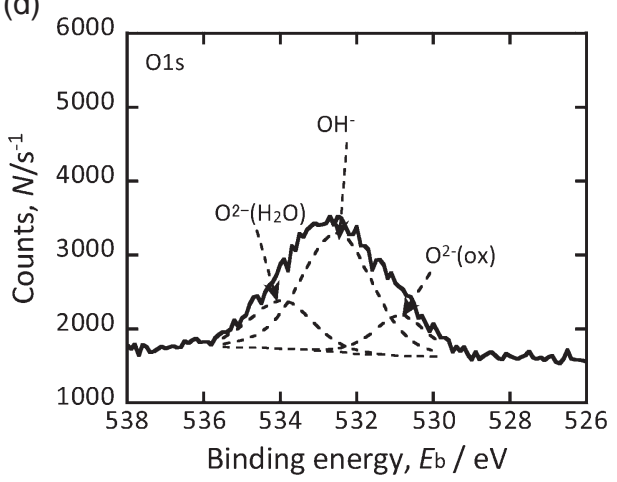

Fig. 4 XPS Ni2 $p_{3 / 2}, \mathrm{Cr} 2 \mathrm{p}_{3 / 2}, \mathrm{Fe} 2 \mathrm{p}_{3 / 2}$ and O1s spectra detected for the passive film formed on Alloy 690 at $200 \mathrm{mV}$ in the borate buffer solution.

the spectra in the lower energy region to the horizontal axis provides the lower band gap energy derived from one layer of passive film and the slope of the spectra. By subtracting the photoelectrochemical response for the component with lower band gap energy from the original photoelectrochemical action spectra, photoelectrochemical action spectra ascribed to the other component with higher band gap energy are obtained at higher photon energy region. Then, the extrapolation of the remaining spectra to the horizontal axis provides the larger component of band gap energy. Details of the separation process were previously reported. ${ }^{17)}$ From the photoelectrochemical action spectra shown in Fig. 3(b), the band gap energies for the passive film formed on Alloy 690 at $200 \mathrm{mV}$ in the borate buffer solution were estimated as approximately $2.3 \mathrm{eV}$ and $3.5 \mathrm{eV}$. The band gap energies obtained were independent of film formation potential and measuring potential. Similar band gap energies were obtained also for passive films formed on the other specimens as Alloy 600 and Ni-30Cr. Figure 5 shows variation in the slope of the photoelectrochemical action spectrum, $C$, for passive films formed on Alloy 690, Alloy 600 and $\mathrm{Ni}-30 \mathrm{Cr}$ in the borate buffer solution. It is clear that $C$ values for the component with lower band gap energy increases from negative to positive with increasing applied potential although the values are very small. On the other hand, $C$ values for the component with the band gap energy of $3.5 \mathrm{eV}$ are negative for all applied potentials and their absolute values increases with decreasing applied potential. Furthermore the $C$ value obtained on $\mathrm{Ni}-30 \mathrm{Cr}$ is quite different from those on Alloy 600 and Alloy 690. Figure 6 also shows variation in the slope of the photoelectrochemical action spectrum, $C$, for passive films formed on the specimens in the sulfuric acid solution. It

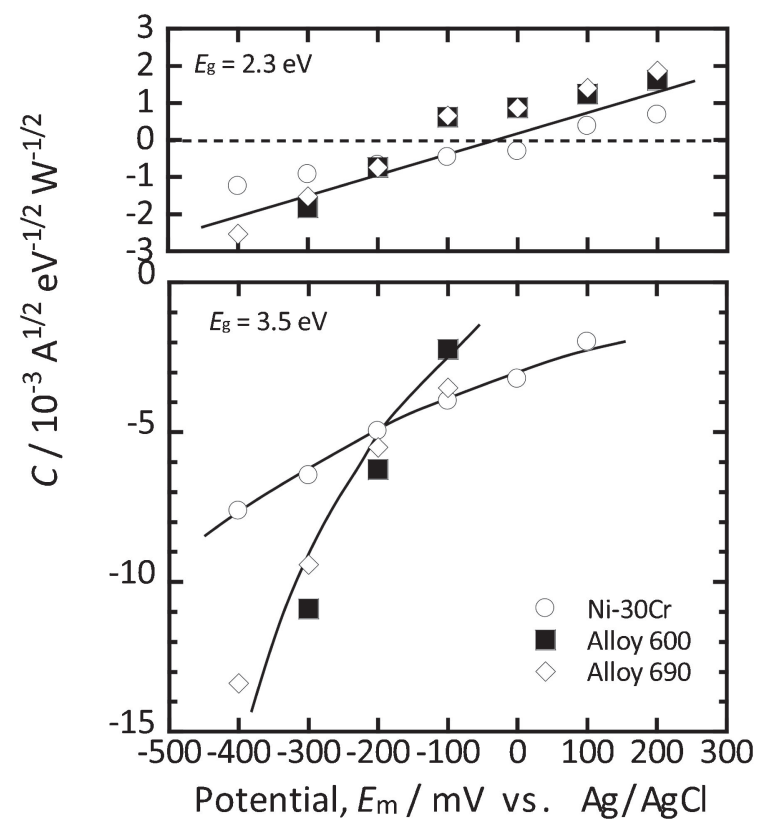

Fig. 5 Variations in the slopes of the photoelectrochemical action spectra, $C$, of passive films formed in the borate buffer solution.

is clear that also in the case of sulfuric acid solution, $C$ values for the component with the band gap energy of $2.3 \mathrm{eV}$ gradually increases from negative to positive with the increase of potential whereas negative $C$ values estimated for the component with larger band gap energy increase the absolute value with decreasing potential, which is in line with the case of the borate buffer solution mentioned above. However, the $C$ value obtained for the component with larger 


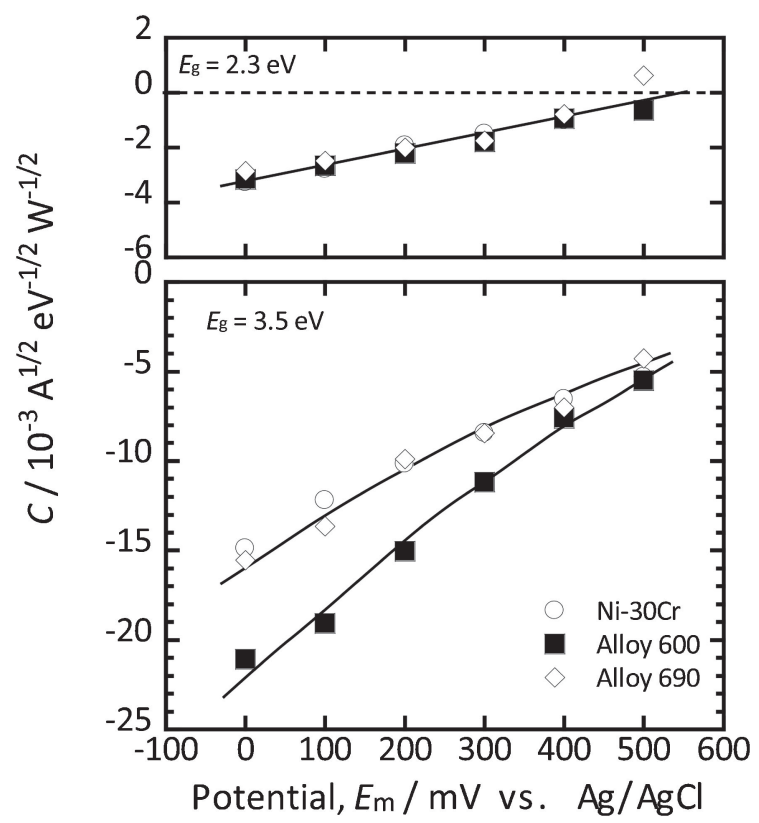

Fig. 6 Variations in the slopes of the photoelectrochemical action spectrum, $C$, of passive films formed in the sulfuric acid solution.

band gap energy on $\mathrm{Ni}-30 \mathrm{Cr}$ in the sulfuric acid solution is very similar to that on Alloy 690 although $C$ values for the components with lower band gap energy of $2.3 \mathrm{eV}$ are quite similar for all specimens. These results indicate that $C$ value can be changed depending on solution used for experiment.

\section{Discussion}

In the present work electronic properties of passive films formed on Alloy 600 and Alloy 690 in the borate buffer and sulfuric acid solutions were examined by the photoelectrochemical response. The obtained photoelectrochemical action spectra were separated into two components, providing two band gap energies of $2.3 \mathrm{eV}$ and $3.5 \mathrm{eV}$ for passive films formed on Alloy 600 and Alloy 690 as well as Ni-30Cr in the solutions. As described above, the passive films formed on the Ni-based alloys in the solutions consist mainly of hydroxide and oxide layers of $\mathrm{Ni}$ and $\mathrm{Cr}$. Referring from the band gap energies reported previously for oxide and hydroxide layers on $\mathrm{Ni}$ and $\mathrm{Cr},{ }^{11,15,16,22,26)}$ the band gap energies estimated in the present work for the passive films on Alloy 600, Alloy 690 and Ni-30Cr in both solutions can be attributed to hydroxide and oxide layers in the passive films on the Ni-based alloys, that is, the band gap energy of $2.3 \mathrm{eV}$ is attributed to the hydroxide layer whereas the band gap energy of $3.5 \mathrm{eV}$ is from the oxide layer. The potential dependence of the slope of the photoelectrochemical action spectrum shown in Figs. 5 and 6 was quite similar to those obtained for passive films formed on $\mathrm{Fe}-18 \mathrm{Cr}$ in a sulfuric acid solution, ${ }^{19)}$ that is, the $C$ values for oxide layer were negative for all applied potentials and the $C$ values increase with decreasing applied potential whereas the $C$ values for hydroxide layer are very small compared to those for oxide layer and increase from negative to positive with increasing applied potential. Therefore the electronic band structure of passive films formed on Alloy 600, Alloy 690 and Ni-30Cr is

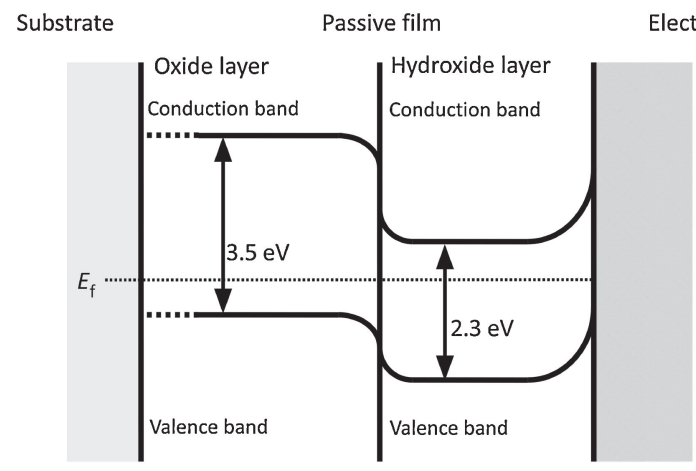

Fig. 7 A band structure model for passive films formed on Ni-based alloys. " $E_{\mathrm{f}}$ " in the illustration indicates Felmi energy level.

considered to be similar to that proposed for stainless steel passive films on the Ni-based alloys consist of p-type inner oxide layer with the band gap energy of $3.5 \mathrm{eV}$ and n-type outer hydroxide layer with the band gap energy of $2.3 \mathrm{eV}$ as illustrated in Fig. 7.

As described above, the electronic band structure of passive films on the specimens are same. However, a difference can be observed in the amplitude of photocurrent, that is, the $C$ value is different according to specimen, as apparent from Figs. 5 and 6, especially for the inner oxide layers with the band gap energy of $3.5 \mathrm{eV}$. On the other hand, the $C$ value for the hydroxide layers with the band gap energy of $2.3 \mathrm{eV}$ was more or less similar independent of specimen. Therefore, the variation in the $C$ value for the oxide layer is discussed here. This can be related to the chemical composition of the oxide layers in the passive films on the Ni-based alloys. Table 2 presents chemical compositions of passive films examined in the present work. The details on the calculation method for the chemical compositions were previously described in the literature. ${ }^{29,30)}$ As listed in Table 2, the oxide layers in the passive films formed on Alloy 600, Alloy 690 and Ni-30Cr consist mainly of Cr oxide and $\mathrm{Ni}$ oxide in the borate buffer solution as well as the sulfuric acid solution. However, more Fe was incorporated into the oxide layers of the passive films on Alloy 600 and Alloy 690 in the borate buffer solution compared to in the sulfuric acid solution. Therefore, it can be assumed that such enrichment of Fe oxide in the oxide layers affects the photocurrent generation. The similar potential dependence of the $C$ value was obtained for the oxide layer of the passive films formed on Alloy 600 and Alloy 690, which is relatively different from that for the oxide layer on $\mathrm{Ni}-30 \mathrm{Cr}$ that contains no Fe. On the other hand, in the case of the sulfuric acid solution, less Fe was incorporated into the oxide layers on Alloy 600 and Alloy 690 due to the selective dissolution of $\mathrm{Fe}$. As a result, the effect of $\mathrm{Fe}$ in the oxide layer of passive films on the photocurrent generation could be negligible and therefore it is found that the photocurrent generation was mainly determined by $\mathrm{Cr}$ content in the oxide layers of the passive films in the sulfuric acid solution. Therefore, as shown in Fig. 6, the potential dependence of the $C$ value for the oxide layer of the passive film on Alloy 690 was almost same as that for $\mathrm{Ni}-30 \mathrm{Cr}$ as the $\mathrm{Cr}$ content in the oxide layer of the passive film on Alloy 690 is similar to that on $\mathrm{Ni}-30 \mathrm{Cr}$ due to their similar $\mathrm{Cr}$ content in the 
Table 2 Cation fractions in passive films formed on the specimens. "ox" and "hyd" in this table mean data on cation fraction for each element in oxide and hydroxide layers, respectively.

\begin{tabular}{|c|c|c|c|c|c|c|c|}
\hline & & \multicolumn{3}{|c|}{ Oxide layer (inner) } & \multicolumn{3}{|c|}{ Hydroxide layer (outer) } \\
\hline & & $\mathrm{Ni}(\mathrm{ox})$ & $\mathrm{Cr}(\mathrm{ox})$ & $\mathrm{Fe}(\mathrm{ox})$ & Ni(hyd) & Cr(hyd) & $\mathrm{Fe}(\mathrm{hyd})$ \\
\hline \multirow{3}{*}{ Sulfuric acid solution } & $\mathrm{Ni}-30 \mathrm{Cr}$ & 0.040 & 0.960 & - & 0.210 & 0.790 & - \\
\hline & Alloy 600 & 0.115 & 0.698 & 0.187 & 0.197 & 0.571 & 0.232 \\
\hline & Alloy 690 & 0.067 & 0.823 & 0.110 & 0.094 & 0.682 & 0.224 \\
\hline \multirow{3}{*}{ Borate buffer solution } & $\mathrm{Ni}-30 \mathrm{Cr}$ & 0.220 & 0.780 & - & 0.415 & 0.585 & - \\
\hline & Alloy 600 & 0.341 & 0.323 & 0.335 & 0.436 & 0.222 & 0.342 \\
\hline & Alloy 690 & 0.159 & 0.502 & 0.339 & 0.206 & 0.588 & 0.206 \\
\hline
\end{tabular}

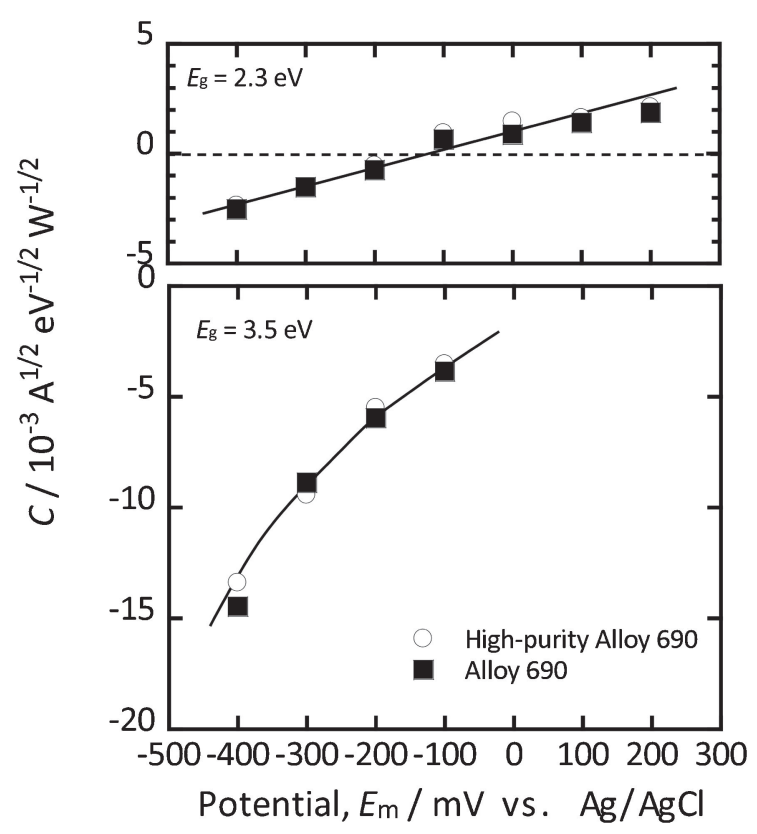

Fig. 8 Variations in the slopes of the photoelectrochemical action spectrum, $C$, of passive films formed on conventional and high-purity Alloy 690 in the borate buffer solution.

substrates and the selective dissolution of Fe from Alloy 690 in the acidic solution. As Alloy 690 contains other impurities as listed in Table 1, their influence on the photocurrent generation in passive film was also examined. Figure 8 shows the potential dependence of the $C$ value for the passive film formed on high-purity Alloy 690 in the borate buffer solution. The chemical composition of the alloy is already presented in Table 1. The $C$ value obtained for the passive film on the high-purity Alloy 690 is totally same as that for the passive film on the conventional Alloy 690, that is, impurities that are included in conventional Alloy 690 do not affect the electronic properties of passive films on Alloy 690. Therefore, it can be concluded that electronic properties of passive films can be affected when a certain amount of impurity is incorporated into passive films.

\section{Conclusions}

We examined passive films formed on nickel-based alloys in borate buffer and sulfuric acid solutions by the photo- electrochemical response and X-ray photoelectron spectroscopy. The results indicated that the passive films formed on the alloys in the solution consist of an inner oxide layer with the bang gap energy of approximately $3.5 \mathrm{eV}$ and a covering hydroxide layer with the bang gap energy of around $2.3 \mathrm{eV}$. The photoelectrochemical response was significantly changed for oxide layer, according to the specimen as well as the solution used. This can be ascribed to the difference in chemical composition of oxide layer where the amount of incorporated $\mathrm{Fe}$ is significantly different.

\section{Acknowledgements}

The present work is supported by "Priority Assistance of the Formation of Worldwide Renowned Centers of Research - Global COE Program (Center of Excellence for Advanced Structure and Functional Materials Designs)" from the Ministry of Education, Culture, Sports, Science and Technology of Japan.

\section{REFERENCES}

1) G. Okamoto: Corros. Sci. 13 (1973) 471-489.

2) I. Olefjord and H. Fischmeister: Corros. Sci. 15 (1975) 697-707.

3) H.-H. Strehblow and B. Titze: Electrochim. Acta 25 (1980) 839-850.

4) P. Marcus, I. Olefjord and J. Oudar: Corros. Sci. 24 (1984) 269-278.

5) P. Marcus and J. M. Grimal: Corros. Sci. 33 (1992) 805-814.

6) T. Jabs, P. Borthen and H.-H. Strehblow: J. Electrochem. Soc. 144 (1997) 1231-1243.

7) J. B. Lumsden and R. W. Staehle: Scr. Metall. 6 (1972) 1205-1208.

8) R. W. Revie, B. G. Baker and J. O’M. Bockris: J. Electrochem. Soc. 122 (1975) 1460-1466.

9) M. Seo, R. Saito and N. Sato: J. Electrochem. Soc. 127 (1980) 19091912 .

10) W. E. O’Grady: J. Electrochem. Soc. 127 (1980) 555-563.

11) S. M. Wilhelm and N. Hackerman: J. Electrochem. Soc. 128 (1981) 1668-1674.

12) K. Sugimoto and S. Matsuda: J. Electrochem. Soc. 130 (1983) 23232329.

13) H. Gerischer: Corros. Sci. 31 (1990) 81-88.

14) C. Sunseri, S. Piazza, D. Paola and D. Quarto: J. Electrochem. Soc. 134 (1987) 2410-2416.

15) F. Di Quarto, S. Piazza and C. Sunseri: Corros. Sci. 31 (1990) 721-726.

16) C. Sunseri, S. Piazza and F. Di Quarto: Mater. Sci. Forum 185-188 (1995) 435-446.

17) S. Fujimoto, O. Chihara and T. Shibata: Mater. Sci. Forum 289-292 (1998) 989-996.

18) M. Da Cunha Belo, N. E. Hakiki and M. G. S. Ferreira: Electrochim. Acta 44 (1999) 2473-2481. 
19) H. Tsuchiya, S. Fujimoto, O. Chihara and T. Shibata: Electrochim. Acta 47 (2002) 4357-4366.

20) H. Tsuchiya, S. Fujimoto and T. Shibata: J. Electrochem. Soc. 151 (2004) B39-B44.

21) M. F. Montemor, M. G. S. Ferreira, N. E. Hakiki and M. Da Cunha Belo: Corros. Sci. 42 (2000) 1635-1650.

22) Y. Mito, M. Ueda and T. Ohtsuka: Corros. Sci. 51 (2009) 1540-1544.

23) J. Huang, X. Wu and E.-H. Han: Corros. Sci. 51 (2009) 2976-2982.

24) D.-J. Kim, H.-C. Kwon and H. P. Kim: Corros. Sci. 50 (2008) $1221-$ 1227.

25) B. Ter-Ovanessian, C. Alemany-Dumont and B. Normand: Electro- chim. Acta 133 (2014) 373-381.

26) L. Marchetti, S. Perrin, Y. Wouters, F. Martin and M. Pijolot: Electrochim. Acta 55 (2010) 5384-5392.

27) K. Fushimi, K. Kurauchi, Y. Yamamoto, T. Nakanishi, Y. Hasegawa and T. Ohtsuka: Electrochim. Acta 144 (2014) 56-63.

28) D. A. Shirley: Phys. Rev. B 5 (1972) 4709-4714.

29) S. Fujimoto: Progress in Advanced Structural and Functional Materials Design, ed. by T. Kakeshita, (Springer Japan, 2013) pp. 72-81.

30) S. Fujimoto, S. Kawachi and T. Shibata: J. Japan Inst. Metals 63 (1999) $375-382$. 